(1)

CrossMark

\title{
Anti-alarmins in asthma: targeting the airway epithelium with next-generation biologics
}

\author{
Celeste M. Porsbjerg ${ }^{1}$, Asger Sverrild ${ }^{1}$, Clare M. Lloyd (10², \\ Andrew N. Menzies-Gow ${ }^{3}$ and Elisabeth H. Bel ${ }^{4}$
}

Affiliations: ${ }^{1}$ Dept of Respiratory Medicine, Bispebjerg Hospital, Copenhagen, Denmark. ${ }^{2}$ National Heart and Lung Institute, Imperial College London, London, UK. ${ }^{3}$ Royal Brompton Hospital, London, UK. ${ }^{4}$ Dept of Respiratory Medicine, Amsterdam University Medical Centre, University of Amsterdam, Amsterdam, The Netherlands.

Correspondence: Celeste M. Porsbjerg, Dept of Respiratory Medicine, Bispebjerg Hospital, Bispebjerg Bakke 23, 2400 Copenhagen, Denmark. E-mail: Celeste.PorsbjergAregionh.dk

@ERSpublications

Blocking epithelial alarmins, upstream mediators triggered early in the asthma inflammatory response that orchestrate broad inflammatory effects, is a promising alternative approach to asthma treatment, which may be effective in a broad patient population https://bit.ly/2zqoXAw

Cite this article as: Porsbjerg CM, Sverrild A, Lloyd CM, et al. Anti-alarmins in asthma: targeting the airway epithelium with next-generation biologics. Eur Respir J 2020; 56: 2000260 [https://doi.org/10.1183/ 13993003.00260-2020].

ABSTRACT Monoclonal antibody therapies have significantly improved treatment outcomes for patients with severe asthma; however, a significant disease burden remains. Available biologic treatments, including anti-immunoglobulin (Ig)E, anti-interleukin (IL)-5, anti-IL-5R $\alpha$ and anti-IL-4R $\alpha$, reduce exacerbation rates in study populations by approximately $50 \%$ only. Furthermore, there are currently no effective treatments for patients with severe, type 2-low asthma. Existing biologics target immunological pathways that are downstream in the type 2 inflammatory cascade, which may explain why exacerbations are only partly abrogated. For example, type 2 airway inflammation results from several inflammatory signals in addition to IL-5. Clinically, this can be observed in how fractional exhaled nitric oxide $\left(F_{\mathrm{eNO}}\right)$, which is driven by IL-13, may remain unchanged during anti-IL-5 treatment despite reduction in eosinophils, and how eosinophils may remain unchanged during anti-IL-4R $\alpha$ treatment despite reduction in $F_{\text {eNO }}$. The broad inflammatory response involving cytokines including IL-4, IL-5 and IL-13 that ultimately results in the classic features of exacerbations (eosinophilic inflammation, mucus production and bronchospasm) is initiated by release of "alarmins" thymic stromal lymphopoietin (TSLP), IL-33 and IL-25 from the airway epithelium in response to triggers. The central, upstream role of these epithelial cytokines has identified them as strong potential therapeutic targets to prevent exacerbations and improve lung function in patients with type 2-high and type 2-low asthma. This article describes the effects of alarmins and discusses the potential role of anti-alarmins in the context of existing biologics. Clinical phenotypes of patients who may benefit from these treatments are also discussed, including how biomarkers may help identify potential responders. 


\section{Introduction}

Asthma is a chronic, inflammatory disease of the airways that affects over 300 million individuals worldwide [1]. Approximately 5-10\% of these individuals have severe asthma $[2,3]$. Despite a regimen of multiple maintenance medications, patients with severe asthma carry a substantial burden of disease, experiencing frequent exacerbations and having high rates of healthcare resource use, which are associated with substantial costs $[4,5]$. These patients also have severely impaired health-related quality of life, with numerous aspects adversely affected, including sleep, work, study, exercise and daily activities. Comorbidities in severe asthma are common and include chronic rhinosinusitis, nasal polyposis and atopic dermatitis [6].

The management of severe asthma is a considerable challenge, particularly in patients with recurrent exacerbations, because these patients are often receiving maximal inhaled therapy and may require regular oral corticosteroid (OCS) treatment [7]. Until relatively recently, there were few alternatives to regular OCS in these patients, but the advent of monoclonal antibody therapies provided physicians with important additional options for exacerbation prevention, potentially enabling tapering or discontinuation of OCS [8]. These biologic therapies target the signalling pathways involved in type 2 inflammation, mediated by the action of cytokines, such as interleukin (IL)-4, IL-5 and IL-13. These pathways play an important role in the pathogenesis of type 2-high asthma, which is characterised by high levels of type 2 inflammation, as measured using biomarkers such as blood and sputum eosinophils, and fractional exhaled nitric oxide $\left(F_{\text {eNO }}\right)$ [9]. However, currently available biologic therapies, comprising anti-immunoglobulin (Ig)E, anti-IL-5, anti-IL-5R $\alpha$ and anti-IL-4R $\alpha$ monoclonal antibodies (the latter blocking the IL-4 and IL-13 pathways), decrease exacerbation rates in study populations by only $\sim 50 \%$ [10-12]. Furthermore, anti-IgE, anti-IL-5 and anti-IL-4/IL-13 therapies typically produce variable improvements in lung function and symptom scores, although some individuals may experience clinically significant improvements [10-13]. An explanation for this lack of complete efficacy may be that each therapy targets only some of the elements of the pathways that regulate type 2 inflammation, leaving other elements of the disease pathophysiology untreated; hence, there is a need for treatments with broader effects on type 2 inflammation. Furthermore, we urgently need treatments for patients with type 2-low asthma, in whom the disease shows less involvement of type 2 pathways [9]. Defining and therefore diagnosing type 2-low asthma is made challenging by the lack of reliable biomarkers. Although sputum neutrophilia (in the absence of sputum eosinophilia) may be present in some patients, diagnosis of this asthma subtype is generally inferred from the absence of type 2 biomarker elevation [14]. Related to this, it is important to emphasise that the use of corticosteroids, as recommended in patients with severe asthma, reduces eosinophil counts and can therefore confound type 2-low classification [15]. For the purposes of this paper, we define type 2-low asthma as asthma with low type 2 biomarker levels.

\section{Inflammatory pathways in asthma}

Within the type 2-high subtype, the major pathways involved in airway inflammation (i.e. IL-4, IL-13 and IL-5 signalling) are driven by T helper type 2 (Th2) cells and type 2 innate lymphoid cells (ILC2) [16]. IL-4 signalling is central to B cell class switching and triggers the release of IgE from B cells, resulting in airway hyperresponsiveness among other effects [17]. IL-13 is believed to be a central regulator of IgE synthesis, as well as of mucus hypersecretion, airway hyperresponsiveness and fibrosis (an element of airway remodelling) $[18,19]$. IL-5 is a key effector of eosinophilic inflammation [20], which results in airway remodelling $[21,22]$.

The different type 2 pathways have varying prominence across clinical phenotypes, resulting in distinct patterns of inflammatory biomarkers [23]. For instance, patients with early-onset allergic asthma have high serum levels of allergen-specific IgE and total IgE, and elevated $F_{\text {eNO }}[23,24]$, which is characteristic of inflammation predominantly driven by Th2 cells and/or IL-4/IL-13. In contrast, late-/adult-onset eosinophilic asthma is a particularly severe form of the disease characterised by higher levels of sputum eosinophils than early onset asthma [25], as well as elevated blood eosinophils [24, 26], related predominantly to IL-5-mediated inflammation. Elevated $F_{\mathrm{eNO}}$, driven by IL-13, is also characteristic of this phenotype [27]. In the clinic, it may be observed that $F_{\text {eNO }}$ remains high in these patients during anti-IL-5 treatment, despite the reduction in eosinophils $[28,29]$. Similarly, anti-IL-4R $\alpha$ treatment can reduce $F_{\mathrm{eNO}}$, but transient eosinophilia may be observed [8]; caution should therefore be taken when considering anti-IL-4R $\alpha$ for patients with a history of hypereosinophilic conditions [30]. Alternatively, mixed granulocytic asthma may be present, in which there are elevated levels of both eosinophils and neutrophils [24]. In adults with non-eosinophilic asthma, within the type 2-low subtype, there is airway inflammation with a lack of eosinophils [24]. Instead, the dominant inflammatory cell types in blood and sputum may include neutrophils, or there may be very few inflammatory cells, termed paucigranulocytic inflammation $[24,31,32]$. 
Viruses, allergens, cigarette smoke and pollution

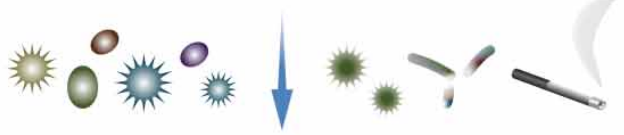

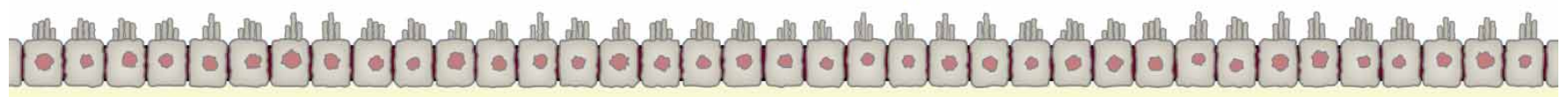

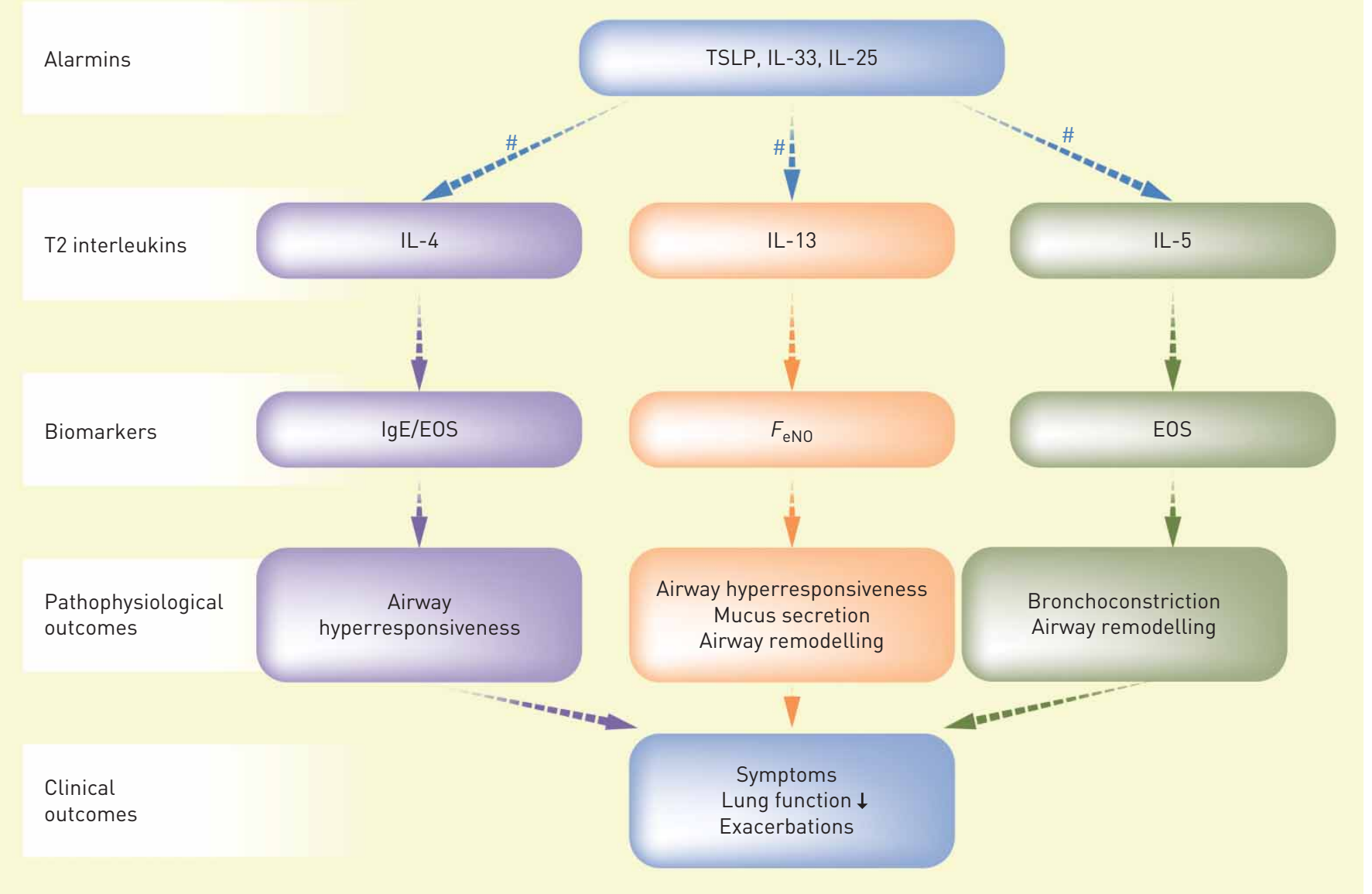

FIGURE 1 Role of the alarmins in driving type 2 inflammation, biomarkers and clinical outcomes in asthma. EOS: eosinophils; $F_{\text {eNo: }}$ fractional exhaled nitric oxide; IgE: immunoglobulin E; IL: interleukin; ILC2: type 2 innate lymphoid cell; Th2: type 2 T helper cell; TSLP: thymic stromal lymphopoietin. \#: Released from Th2 cells and ILC2s.

Our understanding of the role of the airway epithelium in driving asthma exacerbations has advanced considerably in recent years, from a simple passive barrier function to an immunologically active front-line, initiating early responses to external triggers and orchestrating the resulting inflammatory cascade. As the interface between the external environment and the tissue of the bronchi, the airway epithelium is in constant contact with an array of stimuli, such as infectious agents, environmental allergens and atmospheric pollutants. The epithelium has been shown to mediate complex inflammatory processes in response to these allergic and non-allergic triggers, including the release of a trio of epithelial cytokines, known as "alarmins" [33]. The alarmins thymic stromal lymphopoietin (TSLP), IL-33 and IL-25 instigate inflammatory responses via numerous downstream pathways, including type 2 (IL-4, IL-13 and IL-5) (figure 1) and others such as Th1- or Th17- (IL-17) driven pathways, resulting in various pathophysiologic outcomes that may lead to asthma symptoms and exacerbations [33]. It should be noted here that the term "alarmin" is not limited to these three epithelial cytokines; other proteins produced in response to microbial invasion or tissue injury may also be referred to as alarmins [34].

\section{Understanding the pathophysiology of acute exacerbations: a path to better treatments?}

To improve management options for patients with asthma, we require a better understanding of the mechanisms driving acute asthma exacerbations. Exacerbations are characterised by increasing symptoms, 
including dyspnoea, wheezing, cough and sputum, owing to increased inflammation and bronchospasm. They may be triggered by exposure to environmental allergens or pollutants but are most often provoked by viral infection (most commonly with rhinovirus) [35-37]. As with the overall patterns of chronic inflammation in patients with asthma described above, the pattern of inflammatory pathways activated during acute exacerbations is also heterogeneous, with some patients having eosinophilic inflammation and others having neutrophilic, mixed or paucigranulocytic inflammation [38-40]. In patients with eosinophilic exacerbations, inflammation appears to be driven by multiple, simultaneously activated type 2 pathways (e.g. both IL-5 and IL-4/IL-13). This can be inferred from human allergen challenge studies that show increases in airway IL-5, IL-4 and IL-13 levels during virus-induced asthma exacerbations [41, 42], as well as proteomic data showing elevated sputum levels of both IL-5 and IL-13 in patients with eosinophilic asthma [43]. With regard to the considerable proportion of patients who have non-eosinophilic, neutrophilic inflammation in relation to an exacerbation [24], less is understood about the pathways involved in non-eosinophilic exacerbations, although they may involve IL-17 and IL-23 [44, 45]. There is also evidence of airway microbiome involvement because subclinical infection by particular bacterial species is associated with neutrophilic, steroid-resistant asthma [46, 47].

Potential associations between an inflammatory asthma phenotype and specific exacerbation triggers are also poorly understood. Virus-induced exacerbations are more common in patients with allergic asthma [35], as well as in patients with elevated $F_{\mathrm{eNO}}$ or blood eosinophils [48], suggesting a link between virus-induced exacerbations and type 2 inflammation. Eosinophils, activated by IL-5, drive pathophysiology in exacerbating and stable eosinophilic asthma. Eosinophils have recently been found to play a role in the defence against viruses. However, in patients with asthma, the degree to which eosinophils can carry out this role correlates with disease severity [49], explaining why patients with asthma and high eosinophil counts still experience virus-induced exacerbations. This may also explain the lack of efficacy of anti-IL-5 therapy in mild asthma. For example, in a virus-challenge study in patients with mild asthma, anti-IL-5 altered elements of the immune response, including B lymphocytes, macrophages and neutrophils, as well as attenuated eosinophils, but there were no associated improvements in lung function [50]. Less is known about potential links between asthma phenotypes and other associated exacerbation triggers.

There is a pressing need for better understanding of the drivers of exacerbations. One route towards achieving this is to study the impact of specific biologics on patient exacerbations with respect to their phenotypes and triggers. A retrospective analysis of exacerbations in a trial in patients with severe eosinophilic asthma receiving anti-IL-5 treatment with mepolizumab found that exacerbations occurring during treatment were associated with a lower induced sputum eosinophil count and less wheezing than those occurring in patients receiving placebo. This was particularly apparent in patients who had not started rescue treatment with corticosteroids, suggesting that exacerbations were less severe with mepolizumab [51]. This finding is corroborated by previous observations that non-eosinophilic exacerbations are less severe than eosinophilic exacerbations [52]. Data from ongoing studies designed to explore the nature of exacerbations in patients being treated with biologics, such as anti-IL-5 monoclonal antibodies [53], are keenly anticipated. With our current limited knowledge, without full appreciation of the heterogeneity of inflammation in acute exacerbations, prescription of treatments targeting specific inflammatory pathways may not effectively prevent all exacerbations. Therefore, targeting multiple inflammatory pathways may be a more effective approach.

\section{Epithelial alarmins: early orchestrators of airway inflammation in acute asthma Role of TSLP and IL-33 in type 2-high asthma}

There is substantial evidence that the alarmins, TSLP and IL-33, play key roles in driving type 2 inflammation in asthma. TSLP levels in bronchial lavage fluid and biopsies are elevated in patients with asthma compared with healthy individuals, and correlate with disease severity (including a negative correlation with lung function defined by forced expiratory volume in $1 \mathrm{~s}\left(\mathrm{FEV}_{1}\right)$ ) [54-57]. In addition, genetic studies of TSLP have identified alleles that are associated with asthma [58]. Similarly, expression levels of IL-33 and its receptor ST2 in serum and bronchial biopsy samples are higher in patients with asthma than in healthy individuals, and positively correlate with disease severity [59-61]. Additionally, patients with allergic and eosinophilic asthma phenotypes have higher serum levels of IL-33 than those with non-allergic and non-eosinophilic phenotypes [62]. Furthermore, in patients with allergic asthma, ST2 expression on eosinophils from blood and sputum is significantly upregulated after allergen inhalation challenge [54]. Higher serum soluble ST2 levels are associated with an increased risk of exacerbations [63]. Certain alleles of IL-33 and its receptor are associated with asthma [58], and a rare IL-33 loss-of-function mutation reduces blood eosinophil counts and protects from asthma [64]. Bronchial allergen challenge directly increases airway expression of all three alarmins (TSLP, IL-33 and IL-25) in patients with allergic asthma, to a degree correlating with the degree of airway obstruction [42]. 
TSLP has been shown to drive various elements of asthma pathophysiology, including airway hyperresponsiveness, mucus overproduction and airway remodelling, via effects triggered downstream. However, it should be noted that not all these elements have been demonstrated conclusively in humans. During type 2 inflammatory responses, TSLP potently activates dendritic cells and induces production of the Th2-attracting chemokines thymus and activation-regulated chemokine (TARC; also known as CCL17) and macrophage-derived chemokine (CCL22). TSLP-activated dendritic cells prime naïve T helper cells to produce various inflammatory mediators, including IL-4, IL-5 and IL-13 [65]. The IL-5 secreted from polarised Th2 cells mediates eosinophilic inflammation via effects on eosinophil recruitment, maturation and survival [66]. When activated, eosinophils release cysteinyl leukotrienes, which are potent bronchoconstrictors [22]. They also induce airway remodelling through airway smooth muscle cell proliferation [21] and the release of mediators, such as transforming growth factor- $\beta$, cationic proteins and cytokines, as well as through interactions with mast cells and epithelial cells [22]. Mast cells also release acute-phase inflammatory mediators, including cysteinyl leukotrienes, histamine and prostaglandins [67]. IL-4 and IL-13 secreted from polarised Th2 cells activate B cells, which produce IgE, resulting in airway hyperresponsiveness [18] and triggering mast cell degranulation, leading to vascular permeability [68]. IL-13 also increases mucus production via goblet cell proliferation, and airway inflammation via increased epithelial production of $F_{\mathrm{eNO}}$ [19]. In addition to Th2 cells, TSLP activates multiple other cell types that release IL-13, including mast cells, basophils and ILC2s [69-71].

Meanwhile, IL-33 is thought to act as a positive regulator of TSLP dendritic cell signalling, initiating and maintaining Th2 cell-mediated inflammatory responses [72]. IL-33 drives airway hyperresponsiveness through IL-13-mediated mast cell-airway smooth muscle crosstalk. Human lung mast cells are stimulated by IL-33 to release histamine and IL-13, resulting in airway constriction [60]. Further roles of IL-33 include promoting mast cell survival and cytokine production [73], eosinophil survival [74], basophil activation, survival and proliferation [71], and increasing IL-13 release from ILC2s [75].

\section{Role of IL-25 in type 2-high asthma}

Of the three alarmins, the actions of IL-25 in patients with asthma are probably the least understood. IL-25 appears to play a key role in allergic inflammation, particularly as a driver of the type 2 inflammatory response during virus-induced asthma exacerbations [76]. Elevated plasma levels of IL-25 are associated with the allergic asthma phenotype [77], and IL-25 concentration in sputum correlates with disease severity [78]. Sputum IL-25 is also increased in atopic versus non-atopic asthma patients [78]. In addition, a rare allele of a component of the IL-25 receptor, IL-17RB, is associated with a reduced incidence of asthma [79].

After inhaled allergen exposure, there is increased expression of IL-25 and its receptor in the bronchial mucosa and dermis (collected by skin biopsy) [80], and in circulating eosinophils and airway dendritic cells $[81,82]$ in patients with allergic asthma. Bronchial epithelial expression of IL-25, but not IL-33 or TSLP, is heterogeneous in patients with asthma. Those expressing high levels of IL-25 have greater airway hyperresponsiveness and remodelling, increased blood and airway eosinophilia, and higher allergen skin-test reactivity, with elevated IgE, than those expressing low levels of IL-25 [83]. Aside from epithelial cells, which form the principal location of all three alarmins, IL-25 release appears to be prominently localised to eosinophils [42]. IL-25 is hypothesised to play a role in allergen-induced trafficking of eosinophil-lineage committed progenitor cells to the airways, and local differentiation, promoting tissue eosinophilia during asthmatic responses [84].

\section{Role of TSLP in type 2-low asthma}

Much less is known about the role of alarmins in type 2-low asthma than in type 2-high asthma; most of our understanding comes from animal studies, with relatively few studies having been conducted in humans or human cells. Of the three alarmins, TSLP has the most compelling evidence for an effect in type 2-low asthma. Anti-TSLP treatment has been shown to reduce exacerbations in patients with severe, uncontrolled asthma and low levels of blood eosinophils and $F_{\mathrm{eNO}}$ (as discussed later) [85]. TSLP is thought to play a role in neutrophilic, type 2-low airway inflammation by activating dendritic cells to induce polarisation of naïv $\mathrm{T}$ cells towards a Th17 phenotype [86]. Th17 polarisation and subsequent IL-17 release promotes neutrophilic inflammation and a non-allergic/non-eosinophilic response [87]. TSLP, but not IL-33 or IL-25, expression in human bronchoalveolar lavage fluid has been shown to correlate most closely with neutrophil infiltration [54]. Nevertheless, future studies are warranted to understand whether there is a broader role for IL-33 in the pathogenesis of type 2-low asthma.

\section{Role of the alarmins in steroid-refractory asthma}

Airway levels of ILC2s are elevated in several phenotypes of asthma, including late-onset eosinophilic asthma [25, 88-90], and may be the key driver of eosinophilic inflammation in these patients. Although individual alarmins do not directly promote proliferation of ILC2s, TSLP promotes the longevity of ILC2s, 
while ILC2 activation is promoted by IL-33, especially when in combination with TSLP (or IL-2) [70]. In a recent, in vitro study of human blood and lung ILC2s from patients with asthma and healthy controls, IL-25- and IL-33-stimulated expression of type 2 cytokines by blood ILC2s was inhibited by steroid treatment; however, TSLP-stimulated type 2 cytokine expression was not [91]. TSLP levels were elevated in bronchoalveolar lavage fluid in patients with asthma versus healthy controls and correlated with the level of steroid resistance. TSLP was shown to induce steroid resistance in the ILC2s [91]. TSLP-initiated type 2 signalling may, therefore, be particularly important in severe asthma patients with steroid-refractory disease and both Th2- and ILC2-driven inflammation. Non-eosinophilic asthma is also associated with steroid resistance [92], linked to high IL-17 and high numbers of neutrophils in these patients, potentially resulting from TSLP signalling (as described above) [44, 87].

\section{Targeting the airway alarmins with biologic therapies}

The inability of current biologic therapies to prevent all asthma exacerbations and the complexity of the inflammatory pathways involved in asthma, provide a strong rationale for finding alternative, more effective means of reducing airway inflammation and thereby preventing exacerbations. The central, upstream role of alarmins makes them attractive potential therapeutic targets in this regard. Current biologics are not disease modifying, and available evidence suggests that asthma severity returns to pre-treatment levels after stopping treatment with them [93, 94]. Blocking alarmins, however, has the potential to inhibit airway hyperresponsiveness and remodelling [95] and produce sustained reductions in disease activity. Biologics targeting the alarmins are now in development, with anti-TSLP and anti-IL-33 therapies having entered clinical trials. The current evidence for the effects of alarmin blockade in humans is summarised in table 1 and figure 2 .

\section{Anti-TSLP}

Tezepelumab, an anti-TSLP human monoclonal antibody that prevents TSLP interacting with its heterodimeric receptor [96], is the anti-alarmin that has reached the furthest stage of clinical development (table 1). In a proof-of-concept allergen challenge study in patients with mild allergic asthma, tezepelumab $700 \mathrm{mg}$ was administered every 4 weeks for 12 weeks, with an inhaled allergen challenge at baseline and after 6 and 12 weeks [96]. FEV 1 reductions post-allergen challenge at week 6 and week 12 were significantly smaller with tezepelumab than with placebo ( $34 \%$ and $46 \%$, respectively). In addition, patients receiving tezepelumab had significant decreases in levels of blood and sputum eosinophils before and after allergen challenge. Similarly, $F_{\mathrm{eNO}}$ levels dropped throughout the study, and post-allergen $F_{\mathrm{eNO}}$ increases were significantly reduced. These results paved the way for a phase $2 b$ study in patients with moderate-to-severe uncontrolled asthma, who were administered tezepelumab $70 \mathrm{mg}$ every 4 weeks, 210 mg every 4 weeks or $280 \mathrm{mg}$ every 2 weeks, or placebo every 2 weeks, over 52 weeks [85]. Tezepelumab was well tolerated and significantly reduced annualised asthma exacerbation rates in the tezepelumab

TABLE 1 Clinical evidence of the effects of thymic stromal lymphopoietin blockade in humans ${ }^{\#}$

Reference Setting Outcomes

[85] Patients with uncontrolled moderate-to-severe asthma (phase $2 b$ study)

[110] Patients with uncontrolled moderate-to-severe asthma (phase $2 \mathrm{~b}$ study)

Patients with mild allergic asthma (phase 1/2a study) after allergen challenge Patients with mild allergic asthma (phase 1/2a
study) after allergen challenge
Reduced exacerbation rate irrespective of baseline blood eosinophil count Increased FEV 1

Reduced blood eosinophils, $F_{\mathrm{eNO}}$, serum IgE throughout the 52-week treatment period

Reduced blood eosinophils, $\mathrm{Fe}_{\mathrm{NO}}$, serum IgE, IL-5, IL-13, periostin and TARC throughout the 52-week treatment period

Reduced exacerbation rate irrespective of baseline blood eosinophils, $F_{\mathrm{eNO}}$, serum IgE, IL-5, IL-13, periostin, TARC

Reduced airway hyperresponsiveness

Reduced bronchoconstriction

Reduced blood and sputum eosinophils

Reduced $F_{\mathrm{eNO}}$

Reduced Th2:Th1 cell ratio in blood

No change in T regulatory cell frequency

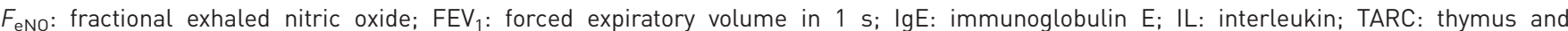
activation-regulated chemokine; Th: T helper. ${ }^{\#}$ : all data are from studies of anti-TSLP human monoclonal antibody treatment. No clinical data evaluating anti-IL-33 or anti-IL-25 treatment have yet been published in peer-reviewed journals. 


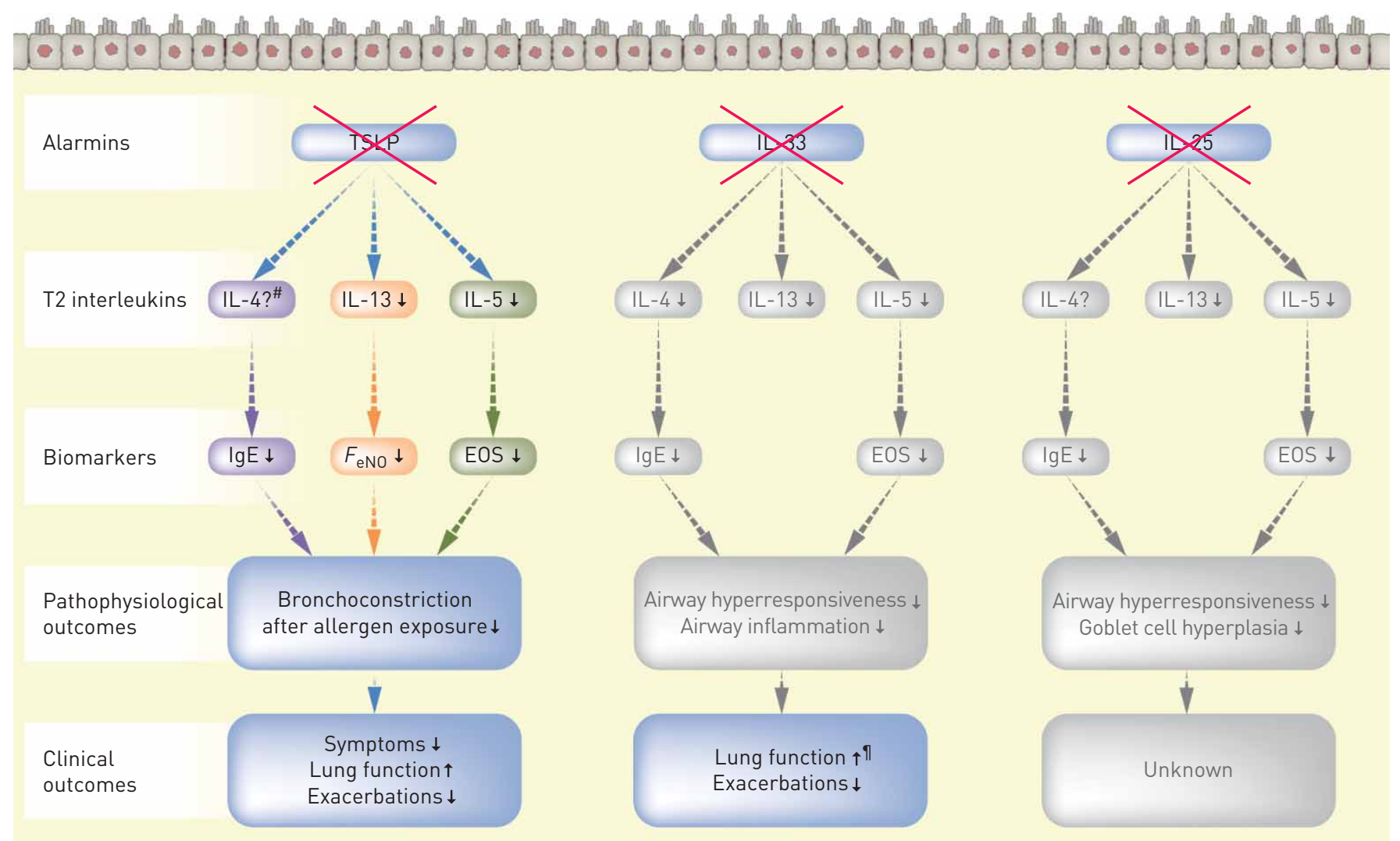

FIGURE 2 Effects of blocking the alarmins on biomarkers and clinical outcomes in asthma. EOS: eosinophils; $F_{\text {eNo: }}$ fractional exhaled nitric oxide; IgE: immunoglobulin E; IL: interleukin; TSLP: thymic stromal lymphopoietin. Grey boxes represent findings from studies in mice, which are yet to be confirmed in humans $[127,128] .{ }^{\#}$ : serum IL-4 was not measured in the tezepelumab phase $2 \mathrm{~b}$ study; ": top-line findings reported from a phase 2a study of anti-IL-33 [101], which are yet to be published in a peer-reviewed journal.

groups versus placebo (by 61\%, 71\% and 66\%, respectively). Similar results were observed regardless of patients' blood eosinophil counts at enrolment. Pre-bronchodilator $\mathrm{FEV}_{1}$ at week 52 improved within the first month of tezepelumab treatment relative to placebo and persisted throughout the study, as did improvements in patient-reported outcomes. In an exploratory analysis of the phase $2 \mathrm{~b}$ data, tezepelumab was found to reduce annualised asthma exacerbation rates irrespective of patients' baseline blood eosinophil count, level of $F_{\mathrm{eNO}}$, or serum levels of IgE, IL-5, IL-13, periostin or TARC [97]. In addition, levels of these proinflammatory biomarkers and cytokines were reduced as early as 4 weeks after treatment initiation; the reductions were then maintained throughout the 52-week treatment period.

Taken together, these findings indicate that tezepelumab has broad inhibitory effects on multiple type 2 inflammatory mediators of asthma, suggesting that anti-TSLP treatment may be beneficial in patients with a range of inflammatory phenotypes (table 2). As described above, because TSLP is postulated to initiate type 2 inflammation via both Th2 and ILC2 cells, blocking TSLP with tezepelumab may effectively reduce inflammation in patients with steroid-refractory asthma. Furthermore, the observation that tezepelumab was efficacious in patients with low blood eosinophils and low $F_{\mathrm{eNO}}$ indicates that it may be effective in the type 2-low asthma subtype. By contrast, the existing biologics targeting type 2 pathways, such as anti-IL-4R and anti-IL-5, have shown less efficacy in patients with low versus high eosinophil counts [98100]. Whether tezepelumab is effective in patients with type 2-low asthma is yet to be confirmed and will depend on how type 2-low asthma is defined in terms of biomarkers in future clinical studies.

Several clinical investigations assessing the efficacy and safety of tezepelumab in different patient populations are ongoing, with results yet to be published (table 3). Two phase 2 (NCT02698501 and NCT03688074) and two phase 3 studies are ongoing: one investigating the efficacy and safety of tezepelumab in reducing OCS use in adults with OCS-dependent asthma (NCT03406078), and one investigating the efficacy and safety of tezepelumab in adults and adolescents with severe, uncontrolled asthma (NCT03347279).

In addition to tezepelumab, CSJ117, an anti-TSLP monoclonal antibody fragment delivered by inhalation, is in phase 1 development (NCT03138811), with results not yet published. 
TABLE 2 Effects on type 2 inflammatory biomarkers, and oral corticosteroid (OCS)-sparing potential, of anti-thymic stromal lymphopoietin (TSLP) and approved biologic therapies for asthma

\begin{tabular}{|c|c|c|c|c|c|}
\hline \multirow[t]{2}{*}{ Biologic } & \multicolumn{4}{|c|}{ Effect on inflammatory biomarker } & \multirow[t]{2}{*}{ OCS-sparing data } \\
\hline & $F_{\mathrm{eNO}}$ & $\lg E$ & $\begin{array}{c}\text { Blood } \\
\text { eosinophils }\end{array}$ & $\begin{array}{c}\text { Sputum } \\
\text { eosinophils }\end{array}$ & \\
\hline $\begin{array}{l}\text { Dupilumab } \\
\text { (anti-IL-4/IL-13) }\end{array}$ & $\downarrow[100]$ & $\downarrow[100]$ & $\uparrow[100]$ & $\begin{array}{l}\text { Insufficient } \\
\text { data [112] }\end{array}$ & $\begin{array}{c}\sim 70 \% \text { reduction in daily OCS dosage (versus } \sim 42 \% \\
\text { with placebo); } 52 \% \text { of patients achieved complete OCS } \\
\text { weaning (placebo, } 29 \% \text { ) [113] }\end{array}$ \\
\hline $\begin{array}{l}\text { Omalizumab } \\
\text { (anti-lgE) }\end{array}$ & $\begin{array}{l}\text { Minimal } \\
\text { effects [114] }\end{array}$ & $\downarrow[115]$ & $\begin{array}{l}\text { Minimal } \\
\text { effects [116] }\end{array}$ & $\downarrow[117]$ & $\begin{array}{l}\text { No RCT data; real-world data indicates reduction in } \\
\text { OCS use [118] }\end{array}$ \\
\hline $\begin{array}{l}\text { Mepolizumab } \\
\text { (anti-IL-5] }\end{array}$ & $\begin{array}{c}\text { No effect } \\
\text { [119] }\end{array}$ & No effect ${ }^{\#}$ & $\downarrow[119,120]$ & $\downarrow[119,120]$ & $\begin{array}{c}\text { 50\% reduction in daily OCS dosage (versus } 0 \% \text { with } \\
\text { placebo); } 14 \% \text { of patients achieved complete OCS } \\
\text { weaning (placebo, 8\%) [121] }\end{array}$ \\
\hline
\end{tabular}

$F_{\mathrm{eNO}}$ : fractional exhaled nitric oxide; IgE: immunoglobulin $\mathrm{E}$; IL: interleukin; OCS, oral corticosteroid; RCT: randomised controlled trial. \#: observed in clinical practice; १": expected based on observations with other anti-IL-5 biologics.

\section{Anti-IL-33}

Monoclonal antibodies targeting IL-33 or ST2 are in clinical development, with several in phase 2 trials (table 3), but results from these studies have not yet been published in peer-reviewed journals. AMG282 (RG6149/MSTT1041A) is being evaluated in a 12-month phase $2 \mathrm{~b}$ study in patients with uncontrolled, severe asthma, with the primary outcome being the rate of exacerbations (NCT02918019). REGN3500 (SAR440340) was recently trialled alone and in combination with dupilumab in a 12-week phase 2a study in patients with moderate-to-severe asthma (NCT03387852). Top-line findings from this study are available but are yet to be published in a peer-reviewed journal. Although the anti-IL-33 antibody met its primary endpoint of reducing the incidence of "loss of asthma control" events compared with placebo, outcomes were no better than those seen with dupilumab monotherapy. In addition, combined REGN3500 and dupilumab did not demonstrate increased benefit compared with dupilumab alone [101]. Two earlier phase 1 studies of REGN3500 have also been completed (NCT03112577, NCT02999711). Etokimab (ANB020) has recently been evaluated in a proof-of-concept phase 2a study in adults with severe eosinophilic asthma, including a primary outcome of change in blood eosinophil count (NCT03469934). GSK3772847 (CNTO7160) has recently completed a phase 2a study in patients with moderately severe asthma, with the primary outcome being loss of asthma control events at week 16 (NCT03207243). A 28-week phase 2 b study in patients with moderate-to-severe asthma with allergic fungal airway disease is ongoing, with primary outcomes of change from baseline in blood eosinophil count and $F_{\mathrm{eNO}}$ levels at week 13 (NCT03393806).

\section{Anti-IL-25}

Although IL-25 appears to play a key role in allergic inflammation, particularly during virus-induced asthma exacerbations (as described above), no clinical studies of anti-IL-25 antibodies are in progress.

\section{Patients who may benefit from anti-alarmin treatment}

From a clinical perspective, there are several groups of patients who may benefit from the potentially broad anti-inflammatory effects of anti-alarmins, such as those with multiple activated type 2 pathways. For example, in patients with allergic eosinophilic asthma, it may be difficult to discern whether exacerbations are primarily driven by allergen exposure or are related to eosinophilic inflammation. The ability to target pathways driving both IgE and eosinophils with a single treatment may be a more attractive option than anti-IgE or anti-IL-5. A second example is those patients with non-allergic eosinophilic asthma who have very high $F_{\mathrm{eNO}}$, indicating concomitant activation of both IL-5 and IL-13, 
TABLE 3 Ongoing and recently completed clinical studies of anti-alarmin treatments in patients with asthma

Drug ClinicalTrials.gov Phase Population $\begin{gathered}\text { Con endpoint } \\ \text { identifier }\end{gathered}$

\begin{tabular}{|c|c|c|c|c|}
\hline \multicolumn{5}{|l|}{ Anti-TSLP } \\
\hline \multirow[t]{4}{*}{ Tezepelumab } & NCT02698501 & 2 & Asthma, taking daily ICS & $\begin{array}{l}\text { Decrease in airway hyperresponsiveness to } \\
\text { mannitol }\end{array}$ \\
\hline & NCT03688074 & 2 & Uncontrolled asthma & $\begin{array}{l}\text { Change from baseline in the number of airway } \\
\text { submucosal inflammatory cells }\end{array}$ \\
\hline & NCT03406078 & 3 & Severe, uncontrolled asthma & $\begin{array}{l}\text { Reduction in OCS dose while not losing } \\
\text { asthma control }\end{array}$ \\
\hline & NCT03347279 & 3 & Severe, uncontrolled asthma & Annualised asthma exacerbation rate \\
\hline \multicolumn{5}{|l|}{ Anti-IL-33 } \\
\hline \multirow[t]{2}{*}{$\begin{array}{l}\text { REGN3500 } \\
\text { (SAR440340) }\end{array}$} & NCT02999711 & 1 & Mild-to-moderate asthma & $\begin{array}{l}\text { Number of treatment-emergent adverse } \\
\text { events }\end{array}$ \\
\hline & NCT03112577 & 1 & Mild, allergic asthma & Sputum inflammatory biomarkers \\
\hline $\begin{array}{l}\text { AMG282 (RG6149/ } \\
\text { MSTT1041A) }\end{array}$ & NCT02918019 & $2 b$ & Severe, uncontrolled asthma & Rate of exacerbations \\
\hline (CNTO7160) & NCT03393806 & $2 b$ & $\begin{array}{l}\text { Moderate-to-severe asthma with } \\
\text { allergic fungal airway disease }\end{array}$ & $\begin{array}{l}\text { Blood eosinophil count and } F_{\text {eNo }} \text { levels at } \\
\text { week } 13\end{array}$ \\
\hline
\end{tabular}

$F_{\text {eNO: }}$ fractional exhaled nitric oxide; ICS: inhaled corticosteroids; IL: interleukin; OCS: oral corticosteroids; TSLP: thymic stromal lymphopoietin.

perhaps through activation of ILC2 cells. Although there is presently no evidence available to inform us whether these patients could achieve sufficient control on either anti-IL-5 or anti-IL-4/13, it appears plausible that some need a broader suppression of the type 2 pathways. Thirdly, patients with severe eosinophilic asthma and comorbidities, such as nasal polyposis or atopic dermatitis, that do not improve with current biologic therapies may also have co-activation of cells releasing both IL-5 and IL-4/IL-13. This suggests that these patients may benefit from an upstream-targeted treatment. Other patients who may benefit from anti-alarmin therapy could include those with predominantly mast cell-driven disease or those with no clear pattern of type 2 inflammation.

\section{Outstanding questions regarding anti-alarmins}

Although tezepelumab has thus far been found to be well tolerated in clinical studies, questions remain regarding the long-term safety of blocking the alarmins and the benefit-risk of anti-alarmin treatment compared with that of cumulative OCS exposure, for which the side effects are well known and can lead to significant morbidity [102]. One area for potential exploration is the antimicrobial activity of TSLP, in particular, the short form of TSLP (sfTSLP), which has been shown to exert potent antimicrobial activity [103]. It is therefore conceivable that blocking sfTSLP could result in a greater risk of infection.

Upregulation of alarmins has been implicated in a number of human cancers, although their role is controversial; in some neoplasms, TSLP appears to play a pro-tumorigenic role, while in others, TSLP seems to be protective [104]. As such, further studies are required to determine the precise role of TSLP in human cancer. Similarly, further studies are warranted to determine the effect of blocking IL-33 on cardiovascular disease, because IL-33 is thought to be cardioprotective [105].

An additional and important question is whether suppressing one alarmin alone is sufficient to suppress all inflammatory pathways induced by the alarmins. In this context, it is clearly important to understand the potential interactions between the alarmins and the relative importance of each alarmin in inflammatory responses. Using chronic models of helminth infection and type 2 cytokine-driven lung inflammation, targeting all three alarmins (TSLP, IL-25 and IL-33) was found to be more efficacious than blocking a single alarmin alone [106]. Furthermore, disruption of all three mediators in a model of chronic house dust mite-induced allergic lung inflammation resulted in reduced inflammation, mucus production and lung remodelling. The authors concluded that the results of these studies suggest redundant roles for TSLP, 
IL-25 and IL-33 in the maintenance of type 2 pathology and that these alarmins interact to potentiate their individual effects. An additional study found that both IL-33 and TSLP were required for epithelial cell IL-25 expression, mucus metaplasia and ILC2 expansion after early-life rhinovirus infection in mice [107]. The authors concluded that generation of mucus metaplasia involves a complex interplay among IL-25, IL-33 and TSLP. Nevertheless, blocking the TSLP pathway alone appears sufficient to suppress inflammation, as shown so far in the tezepelumab studies, albeit other anti-TSLP biologics such as RG7258 were not successfully developed into asthma therapies [108]. To date, no clinical studies have investigated the potential interactions between alarmins in patients with asthma, but it would be of importance to understand such interactions, both in terms of effects on the efficacy and safety of these treatments.

Of further interest is the mechanism by which anti-alarmins prevent exacerbations. It has been postulated that omalizumab exerts its beneficial effects through restoration of innate antiviral immunity in plasmacytoid dendritic cells and through increasing the release of interferon- $\alpha$ on rhinovirus exposure, which is deficient in the presence of high $\operatorname{IgE}$ [109]. It is unknown whether anti-alarmins have an effect on antiviral immunity, and it could be speculated that such a mechanism may result in treatment benefit for patients with either type 2-high or type 2-low asthma. Mechanistic studies are required to determine how these new treatments work.

\section{Conclusions and future perspective}

Monoclonal antibody therapies represent a new treatment era in severe asthma, offering patients who are exacerbating despite high-dose anti-inflammatory treatment a safer and more effective alternative option. However, because blocking specific type 2 pathways with currently available biologics fails to prevent all exacerbations, a significant burden of disease remains. This limitation of current biologics may be a consequence of blocking pathways downstream in the immunological cascade, leaving others still active, as the partial suppression of type 2 pathways may be insufficient to abrogate exacerbations in some patients. Blocking alarmins, upstream mediators triggered early in the inflammatory response that orchestrate broad type 2 inflammatory effects, is a promising alternative approach that may be effective in a broader patient population.

Further research into the role of alarmins in asthma pathogenesis will improve our understanding of disease severity and the heterogeneity in response to current treatments. This research should include studies of differences between the roles of the alarmins in the different clinical phenotypes of severe asthma, such as late-onset eosinophilic asthma versus early onset allergic asthma, as well as their relationship with comorbidities such as atopic dermatitis and nasal polyposis. Understanding the clinical effect of each alarmin in patients with specific phenotypes, and in patients with a combination of asthma, atopic dermatitis and nasal polyposis, will be crucial not only for informing our ability to correctly select the appropriate anti-alarmin as a treatment, but also to our understanding of disease mechanisms in asthma. In addition to this, more studies in which exacerbations are phenotyped are also required to help to identify which types of exacerbation are reduced or otherwise by a given biologic treatment. Finally, mechanistic studies in humans are required to improve our understanding of how next-generation biologics work. This will help clinicians to select the most appropriate treatments for their patients.

Acknowledgements: The authors thank Richard Claes of PharmaGenesis London, London, UK for providing medical writing support, which has been funded by AstraZeneca, Cambridge, UK, in accordance with Good Publication Practice 3 (GPP3) guidelines (www.ismpp.org/gpp3).

Conflict of interest: C.M. Porsbjerg reports grants and personal fees for lectures and consultancy from AstraZeneca, Boehringer Ingelheim, GlaxoSmithKline, Icepharma, Janssen, Nigaard, Nopharma, Novartis, Pharmaxis, Roche, Sandoz, Sanofi and Teva, outside the submitted work. A. Sverrild reports personal fees for advisory board work and lectures from AstraZeneca, personal fees for advisory board work from Sanofi Genzyme, personal fees for lectures from Novartis, outside the submitted work. C.M. Lloyd is a Wellcome senior fellow in basic biomedical science (107059/15/Z). A.N. Menzies-Gow reports grants and personal fees for advisory board work, lectures and consultancy from AstraZeneca, personal fees for advisory board work and lectures from Boehringer Ingelheim and Novartis, personal fees for advisory board work from GlaxoSmithKline, personal fees for advisory board work and consultancy from Sanofi and Vectura, personal fees for advisory board work, lectures and meeting attendance from Teva, personal fees for lectures from Roche, outside the submitted work. E.H. Bel reports personal fees from AstraZeneca, Chiesi, GlaxoSmithKline, Novartis, Sanofi, Sterna and Teva, outside the submitted work.

Support statement: This work was supported by AstraZeneca (via funds for medical writing support). Funding information for this article has been deposited with the Crossref Funder Registry.

\section{References}

1 Global Asthma Network. Global Asthma Network. Global Asthma Report 2018. www.globalasthmareport.org/ Date last updated: January 2018. Date last accessed: January 17, 2020. 
Backman H, Jansson SA, Stridsman C, et al. Severe asthma: a population study perspective. Clin Exp Allergy 2019; 49: 819-828.

Chung KF, Wenzel SE, Brozek JL, et al. International ERS/ATS guidelines on definition, evaluation and treatment of severe asthma. Eur Respir J 2014; 43: 343-373.

Chipps BE, Zeiger RS, Borish L, et al. Key findings and clinical implications from The Epidemiology and Natural History of Asthma: Outcomes and Treatment Regimens (TENOR) study. J Allergy Clin Immunol 2012; 130: 332-342.

Zeiger RS, Schatz M, Dalal AA, et al. Utilization and costs of severe uncontrolled asthma in a managed-care setting. J Allergy Clin Immunol Pract 2016; 4: 120-129.

Boulet LP. Influence of comorbid conditions on asthma. Eur Respir J 2009; 33: 897-906.

Ramsahai JM, Hansbro PM, Wark PAB. Mechanisms and management of asthma exacerbations. Am J Respir Crit Care Med 2019; 199: 423-432.

McGregor MC, Krings JG, Nair P, et al. Role of biologics in asthma. Am J Respir Crit Care Med 2019; 199: 433-445.

Fahy JV. Type 2 inflammation in asthma: present in most, absent in many. Nat Rev Immunol 2015; 15: 57-65.

Normansell R, Walker S, Milan SJ, et al. Omalizumab for asthma in adults and children. Cochrane Database Syst Rev 2014; 1: CD003559.

Farne HA, Wilson A, Powell C, et al. Anti-IL5 therapies for asthma. Cochrane Database Syst Rev 2017; 9: CD010834.

Zayed Y, Kheiri B, Banifadel M, et al. Dupilumab safety and efficacy in uncontrolled asthma: a systematic review and meta-analysis of randomized clinical trials. J Asthma 2019: 1110-1119.

Xiong XF, Zhu M, Wu HX, et al. Efficacy and safety of dupilumab for the treatment of uncontrolled asthma: meta-analysis of randomized clinical trials. Respir Res 2019; 20: 108

Fricker M, Heaney LG, Upham JW. Can biomarkers help us hit targets in difficult-to-treat asthma? Respirology 2017; 22: 430-442.

Cowan DC, Cowan JO, Palmay R, et al. Effects of steroid therapy on inflammatory cell subtypes in asthma. Thorax 2010; 65: 384-390.

Gurram RK, Zhu J. Orchestration between ILC2s and Th2 cells in shaping type 2 immune responses. Cell Mol Immunol 2019; 16: 225-235.

Steinke JW, Borish L. Th2 cytokines and asthma. Interleukin-4: its role in the pathogenesis of asthma, and targeting it for asthma treatment with interleukin-4 receptor antagonists. Respir Res 2001; 2: 66-70.

Rael EL, Lockey RF. Interleukin-13 signaling and its role in asthma. World Allergy Organ J 2011; 4: 54-64

Corren J. Role of interleukin-13 in asthma. Curr Allergy Asthma Rep 2013; 13: 415-420.

Garcia G, Taille C, Laveneziana P, et al. Anti-interleukin-5 therapy in severe asthma. Eur Respir Rev 2013; 22: 251-257.

Halwani R, Vazquez-Tello A, Sumi Y, et al. Eosinophils induce airway smooth muscle cell proliferation. J Clin Immunol 2013; 33: 595-604.

Possa SS, Leick EA, Prado CM, et al. Eosinophilic inflammation in allergic asthma. Front Pharmacol 2013; 4: 46. Kim H, Ellis AK, Fischer D, et al. Asthma biomarkers in the age of biologics. Allergy Asthma Clin Immunol 2017; 13: 48.

Carr TF, Zeki AA, Kraft M. Eosinophilic and noneosinophilic asthma. Am J Respir Crit Care Med 2018; 197: $22-37$.

Miranda C, Busacker A, Balzar S, et al. Distinguishing severe asthma phenotypes: role of age at onset and eosinophilic inflammation. J Allergy Clin Immunol 2004; 113: 101-108.

de Groot JC, Storm H, Amelink M, et al. Clinical profile of patients with adult-onset eosinophilic asthma. ERJ Open Res 2016; 2: 00100-2015.

Hirano T, Matsunaga K. Late-onset asthma: current perspectives. J Asthma Allergy 2018; 11: 19-27. 2017; 140: 1509-1518.

Berry A, Busse WW. Biomarkers in asthmatic patients: has their time come to direct treatment? J Allergy Clin Immunol 2016; 137: 1317-1324.

Brooks GD. Updated evaluation of dupilumab in the treatment of asthma: patient selection and reported outcomes. Ther Clin Risk Manag 2020; 16: 181-187.

Tliba O, Panettieri RA Jr. Paucigranulocytic asthma: Uncoupling of airway obstruction from inflammation. I Allergy Clin Immunol 2019; 143: 1287-1294.

Moore WC, Hastie AT, Li X, et al. Sputum neutrophil counts are associated with more severe asthma phenotypes using cluster analysis. J Allergy Clin Immunol 2014; 133: 1557-1563.

Mitchell PD, O’Byrne PM. Epithelial-derived cytokines in asthma. Chest 2017; 151: 1338-1344. 185-194. Murray CS, Simpson A, Custovic A. Allergens, viruses, and asthma exacerbations. Proc Am Thorac Soc 2004; 1: 99-104.

Heymann PW, Carper HT, Murphy DD, et al. Viral infections in relation to age, atopy, and season of admission among children hospitalized for wheezing. J Allergy Clin Immunol 2004; 114: 239-247.

Nicholson KG, Kent J, Ireland DC. Respiratory viruses and exacerbations of asthma in adults. BMJ 1993; 307: 982-986.

Wark PA, Gibson PG. Asthma exacerbations. 3: pathogenesis. Thorax 2006; 61: 909-915. exacerbation. J Allergy Clin Immunol 1995; 95: 843-852.

Wang F, He XY, Baines KJ, et al. Different inflammatory phenotypes in adults and children with acute asthma. Eur Respir J 2011; 38: 567-574.

Jackson DJ, Makrinioti H, Rana BM, et al. IL-33-dependent type 2 inflammation during rhinovirus-induced asthma exacerbations in vivo. Am J Respir Crit Care Med 2014; 190: 1373-1382. 
Wang W, Li Y, Lv Z, et al. Bronchial allergen challenge of patients with atopic asthma triggers an alarmin (IL-33, TSLP, and IL-25) response in the airways epithelium and submucosa. I Immunol 2018; 201: 2221-2231.

43 Schofield JPR, Burg D, Nicholas B, et al. Stratification of asthma phenotypes by airway proteomic signatures. J Allergy Clin Immunol 2019; 144: 70-82.

44 Chesne J, Braza F, Mahay G, et al. IL-17 in severe asthma. Where do we stand? Am J Respir Crit Care Med 2014; 190: 1094-1101.

45 Nakajima H, Hirose K. Role of IL-23 and Th17 cells in airway inflammation in asthma. Immune Netw 2010; 10: $1-4$.

46 Goleva E, Jackson LP, Harris JK, et al. The effects of airway microbiome on corticosteroid responsiveness in asthma. Am J Respir Crit Care Med 2013; 188: 1193-1201.

47 Earl CS, An SQ, Ryan RP. The changing face of asthma and its relation with microbes. Trends Microbiol 2015; 23: 408-418.

48 Bjerregaard A, Laing IA, Backer V, et al. High fractional exhaled nitric oxide and sputum eosinophils are associated with an increased risk of future virus-induced exacerbations: a prospective cohort study. Clin Exp Allergy 2017; 47: 1007-1013.

49 Sabogal Pineros YS, Bal SM, Dijkhuis A, et al. Eosinophils capture viruses, a capacity that is defective in asthma. Allergy 2019; 74: 1898-1909.

50 Sabogal Pineros YS, Bal SM, van de Pol MA, et al. Anti-IL-5 in mild asthma alters rhinovirus-induced macrophage, B-Cell, and neutrophil responses (MATERIAL). A placebo-controlled, double-blind study. Am J Respir Crit Care Med 2019; 199: 508-517.

51 Shrimanker R, Pavord ID, Yancey S, et al. Exacerbations of severe asthma in patients treated with mepolizumab. Eur Respir J 2018; 52: 1801127.

52 Jayaram L, Pizzichini MM, Cook RJ, et al. Determining asthma treatment by monitoring sputum cell counts: effect on exacerbations. Eur Respir J 2006; 27: 483-494.

53 ClinicalTrials.gov. Exploring asthma exacerbations in mepolizumab treated patients. https://clinicaltrials.gov/ct2/ show/NCT03324230 Date last updated: July 10, 2019. Date last accessed: January 20, 2020.

54 Mitchell PD, Salter BM, Oliveria JP, et al. IL-33 and its receptor ST2 after inhaled allergen challenge in allergic asthmatics. Int Arch Allergy Immunol 2018; 176: 133-142.

55 Ying S, O'Connor B, Ratoff J, et al. Thymic stromal lymphopoietin expression is increased in asthmatic airways and correlates with expression of Th2-attracting chemokines and disease severity. J Immunol 2005; 174: 8183-8190.

56 Shikotra A, Choy DF, Ohri CM, et al. Increased expression of immunoreactive thymic stromal lymphopoietin in patients with severe asthma. J Allergy Clin Immunol 2012; 129: 104-111.

57 Ying S, O'Connor B, Ratoff J, et al. Expression and cellular provenance of thymic stromal lymphopoietin and chemokines in patients with severe asthma and chronic obstructive pulmonary disease. J Immunol 2008; 181: 2790-2798.

58 Moffatt MF, Gut IG, Demenais F, et al. A large-scale, consortium-based genomewide association study of asthma. N Engl J Med 2010; 363: 1211-1221.

59 Prefontaine D, Lajoie-Kadoch S, Foley S, et al. Increased expression of IL-33 in severe asthma: evidence of expression by airway smooth muscle cells. J Immunol 2009; 183: 5094-5103.

60 Kaur D, Gomez E, Doe C, et al. IL-33 drives airway hyper-responsiveness through IL-13-mediated mast cell airway smooth muscle crosstalk. Allergy 2015; 70: 556-567.

61 Momen T, Ahanchian H, Reisi M, et al. Comparison of interleukin-33 serum levels in asthmatic patients with a control group and relation with the severity of the disease. Int J Prev Med 2017; 8: 65.

62 Gasiuniene E, Janulaityte I, Zemeckiene Z, et al. Elevated levels of interleukin-33 are associated with allergic and eosinophilic asthma. Scand J Immunol 2019; 89: e12724.

63 Watanabe M, Nakamoto K, Inui T, et al. Serum sST2 levels predict severe exacerbation of asthma. Respir Res 2018; 19: 169 .

64 Smith D, Helgason H, Sulem P, et al. A rare IL33 loss-of-function mutation reduces blood eosinophil counts and protects from asthma. PLoS Genet 2017; 13: e1006659.

65 Soumelis V, Reche PA, Kanzler $\mathrm{H}$, et al. Human epithelial cells trigger dendritic cell mediated allergic inflammation by producing TSLP. Nat Immunol 2002; 3: 673-680.

66 Karlen S, De Boer ML, Lipscombe RJ, et al. Biological and molecular characteristics of interleukin-5 and its receptor. Int Rev Immunol 1998; 16: 227-247.

67 Bradding P, Walls AF, Holgate ST. The role of the mast cell in the pathophysiology of asthma. J Allergy Clin Immunol 2006; 117: 1277-1284.

68 Galli SJ, Tsai M. IgE and mast cells in allergic disease. Nat Med 2012; 18: 693-704.

69 Allakhverdi Z, Comeau MR, Jessup HK, et al. Thymic stromal lymphopoietin is released by human epithelial cells in response to microbes, trauma, or inflammation and potently activates mast cells. J Exp Med 2007; 204: 253-258.

70 Camelo A, Rosignoli G, Ohne Y, et al. IL-33, IL-25, and TSLP induce a distinct phenotypic and activation profile in human type 2 innate lymphoid cells. Blood Adv 2017; 1: 577-589.

71 Salter BM, Oliveria JP, Nusca G, et al. IL-25 and IL-33 induce type 2 inflammation in basophils from subjects with allergic asthma. Respir Res 2016; 17: 5.

72 Murakami-Satsutani N, Ito T, Nakanishi T, et al. IL-33 promotes the induction and maintenance of Th2 immune responses by enhancing the function of OX40 ligand. Allergol Int 2014; 63: 443-455.

73 Iikura M, Suto H, Kajiwara N, et al. IL-33 can promote survival, adhesion and cytokine production in human mast cells. Lab Invest 2007; 87: 971-978.

74 Johnston LK, Bryce PJ. Understanding interleukin 33 and its roles in eosinophil development. Front Med (Lausanne) 2017; 4: 51.

75 Barlow JL, Peel S, Fox J, et al. IL-33 is more potent than IL-25 in provoking IL-13-producing nuocytes (type 2 innate lymphoid cells) and airway contraction. J Allergy Clin Immunol 2013; 132: 933-941.

76 Beale J, Jayaraman A, Jackson DJ, et al. Rhinovirus-induced IL-25 in asthma exacerbation drives type 2 immunity and allergic pulmonary inflammation. Sci Transl Med 2014; 6: $256 \mathrm{ra} 134$. 
Tang W, Smith SG, Beaudin S, et al. IL-25 and IL-25 receptor expression on eosinophils from subjects with allergic asthma. Int Arch Allergy Immunol 2014; 163: 5-10.

Paplinska-Goryca M, Grabczak EM, Dabrowska M, et al. Sputum interleukin-25 correlates with asthma severity: a preliminary study. Postepy Dermatol Alergol 2018; 35: 462-469.

Jung JS, Park BL, Cheong HS, et al. Association of IL-17RB gene polymorphism with asthma. Chest 2009; 135: 1173-1180.

Corrigan CJ, Wang W, Meng Q, et al. Allergen-induced expression of IL-25 and IL-25 receptor in atopic asthmatic airways and late-phase cutaneous responses. J Allergy Clin Immunol 2011; 128: 116-124.

Tang W, Smith SG, Salter B, et al. Allergen-induced increases in interleukin-25 and interleukin-25 receptor expression in mature eosinophils from atopic asthmatics. Int Arch Allergy Immunol 2016; 170: 234-242.

Tworek D, Smith SG, Salter BM, et al. IL-25 receptor expression on airway dendritic cells after allergen challenge in subjects with asthma. Am J Respir Crit Care Med 2016; 193: 957-964.

Cheng D, Xue Z, Yi L, et al. Epithelial interleukin-25 is a key mediator in Th2-high, corticosteroid-responsive asthma. Am J Respir Crit Care Med 2014; 190: 639-648.

Tang W, Smith SG, Du W, et al. Interleukin-25 and eosinophils progenitor cell mobilization in allergic asthma. Clin Transl Allergy 2018; 8: 5.

Corren J, Parnes JR, Wang L, et al. Tezepelumab in adults with uncontrolled asthma. N Engl J Med 2017; 377: 936-946.

Tanaka J, Watanabe N, Kido M, et al. Human TSLP and TLR3 ligands promote differentiation of Th17 cells with a central memory phenotype under Th2-polarizing conditions. Clin Exp Allergy 2009; 39: 89-100.

Gao H, Ying S, Dai Y. Pathological roles of neutrophil-mediated inflammation in asthma and its potential for therapy as a target. J Immunol Res 2017; 2017: 3743048.

Nagakumar P, Denney L, Fleming L, et al. Type 2 innate lymphoid cells in induced sputum from children with severe asthma. J Allergy Clin Immunol 2016; 137: 624-626.

Smith SG, Chen R, Kjarsgaard M, et al. Increased numbers of activated group 2 innate lymphoid cells in the airways of patients with severe asthma and persistent airway eosinophilia. J Allergy Clin Immunol 2016; 137: 75-86.

Phipatanakul W, Mauger DT, Sorkness RL, et al. Effects of age and disease severity on systemic corticosteroid responses in asthma. Am J Respir Crit Care Med 2017; 195: 1439-1448.

Liu S, Verma M, Michalec L, et al. Steroid resistance of airway type 2 innate lymphoid cells from patients with severe asthma: the role of thymic stromal lymphopoietin. J Allergy Clin Immunol 2018; 141: 257-268.

Pavord ID, Brightling CE, Woltmann G, et al. Non-eosinophilic corticosteroid unresponsive asthma. Lancet 1999; 353: 2213-2214.

Ortega H, Lemiere C, Llanos JP, et al. Outcomes following mepolizumab treatment discontinuation: real-world experience from an open-label trial. Allergy Asthma Clin Immunol 2019; 15: 37.

Haldar P, Brightling CE, Singapuri A, et al. Outcomes after cessation of mepolizumab therapy in severe eosinophilic asthma: a 12-month follow-up analysis. J Allergy Clin Immunol 2014; 133: 921-923.

Gauvreau GM, White L, Davis BE. Anti-alarmin approaches entering clinical trials. Curr Opin Pulm Med 2020; 26: 69-76.

Gauvreau GM, O'Byrne PM, Boulet LP, et al. Effects of an anti-TSLP antibody on allergen-induced asthmatic responses. N Engl J Med 2014; 370: 2102-2110.

Corren J, Garcia Gil E, Parnes JR, et al. Tezepelumab treatment effect on annualized rate of exacerbations by baseline biomarkers in uncontrolled severe asthma patients: phase 2b PATHWAY study. Am J Respir Crit Care Med 2019; 199: A2621.

Corren J, Weinstein S, Janka L, et al. Phase 3 study of reslizumab in patients with poorly controlled asthma: Effects across a broad range of eosinophil counts. Chest 2016; 150: 799-810.

Bleecker ER, FitzGerald JM, Chanez P, et al. Efficacy and safety of benralizumab for patients with severe asthma uncontrolled with high-dosage inhaled corticosteroids and long-acting beta2-agonists (SIROCCO): a randomised, multicentre, placebo-controlled phase 3 trial. Lancet 2016; 388: 2115-2127.

Castro M, Corren J, Pavord ID, et al. Dupilumab efficacy and safety in moderate-to-severe uncontrolled asthma. N Engl J Med 2018; 378: 2486-2496.

Sanofi/Regeneron. Regeneron and Sanofi announce positive topline phase 2 results for IL-33 antibody in asthma. https://investor.regeneron.com/news-releases/news-release-details/regeneron-and-sanofi-announce-positive-toplinephase-2-results Date last updated: June 21, 2019. Date last accessed: January 20, 2020.

Bloechliger M, Reinau D, Spoendlin J, et al. Adverse events profile of oral corticosteroids among asthma patients in the UK: cohort study with a nested case-control analysis. Respir Res 2018; 19: 75.

Bjerkan L, Schreurs O, Engen SA, et al. The short form of TSLP is constitutively translated in human keratinocytes and has characteristics of an antimicrobial peptide. Mucosal Immunol 2015; 8: 49-56.

4 Varricchi G, Pecoraro A, Marone G, et al. Thymic stromal lymphopoietin isoforms, inflammatory disorders, and cancer. Front Immunol 2018; 9: 1595.

Chen WY, Tsai TH, Yang JL, et al. Therapeutic strategies for targeting IL-33/ST2 signalling for the treatment of inflammatory diseases. Cell Physiol Biochem 2018; 49: 349-358.

Vannella KM, Ramalingam TR, Borthwick LA, et al. Combinatorial targeting of TSLP, IL-25, and IL-33 in type 2 cytokine-driven inflammation and fibrosis. Sci Transl Med 2016; 8: 337ra365.

Han M, Rajput C, Hong JY, et al. The innate cytokines IL-25, IL-33, and TSLP cooperate in the induction of type 2 innate lymphoid cell expansion and mucous metaplasia in rhinovirus-infected immature mice. J Immunol 2017; 199: 1308-1318.

8 Snell NJ. Discontinued drug projects in the respiratory therapeutic area during 2012. Expert Opin Investig Drugs 2014; 23: 411-415.

Teach SJ, Gill MA, Togias A, et al. Preseasonal treatment with either omalizumab or an inhaled corticosteroid boost to prevent fall asthma exacerbations. J Allergy Clin Immunol 2015; 136: 1476-1485.

0 Pham T-H, Ren P, Parnes JR, et al. Tezepelumab reduces multiple key inflammatory biomarkers in patients with severe, uncontrolled asthma in the phase 2b PATHWAY study. Am J Respir Crit Care Med 2019; 199: A2677. 
111 Baatjes AJ, Smith SG, Dua B, et al. Treatment with anti-OX40L or anti-TSLP does not alter the frequency of T regulatory cells in allergic asthmatics. Allergy 2015; 70: 1505-1508.

112 Wenzel S, Ford L, Pearlman D, et al. Dupilumab in persistent asthma with elevated eosinophil levels. $N$ Engl J Med 2013; 368: 2455-2466.

113 Rabe KF, Nair P, Brusselle G, et al. Efficacy and safety of dupilumab in glucocorticoid-dependent severe asthma. N Engl J Med 2018; 378: 2475-2485.

114 Bhutani M, Yang WH, Hebert J, et al. The real world effect of omalizumab add on therapy for patients with moderate to severe allergic asthma: The ASTERIX Observational study. PLoS One 2017; 12: e0183869.

115 Busse W, Corren J, Lanier BQ, et al. Omalizumab, anti-IgE recombinant humanized monoclonal antibody, for the treatment of severe allergic asthma. J Allergy Clin Immunol 2001; 108: 184-190.

116 Massanari M, Holgate ST, Busse WW, et al. Effect of omalizumab on peripheral blood eosinophilia in allergic asthma. Respir Med 2010; 104: 188-196.

117 Djukanovic R, Wilson SJ, Kraft M, et al. Effects of treatment with anti-immunoglobulin E antibody omalizumab on airway inflammation in allergic asthma. Am J Respir Crit Care Med 2004; 170: 583-593.

118 Molimard M, Buhl R, Niven R, et al. Omalizumab reduces oral corticosteroid use in patients with severe allergic asthma: real-life data. Respir Med 2010; 104: 1381-1385.

119 Pavord ID, Korn S, Howarth P, et al. Mepolizumab for severe eosinophilic asthma (DREAM): a multicentre, double-blind, placebo-controlled trial. Lancet 2012; 380: 651-659.

120 Flood-Page P, Swenson C, Faiferman I, et al. A study to evaluate safety and efficacy of mepolizumab in patients with moderate persistent asthma. Am J Respir Crit Care Med 2007; 176: 1062-1071.

121 Bel EH, Wenzel SE, Thompson PJ, et al. Oral glucocorticoid-sparing effect of mepolizumab in eosinophilic asthma. N Engl J Med 2014; 371: 1189-1197.

122 Castro M, Zangrilli J, Wechsler ME, et al. Reslizumab for inadequately controlled asthma with elevated blood eosinophil counts: results from two multicentre, parallel, double-blind, randomised, placebo-controlled, phase 3 trials. Lancet Respir Med 2015; 3: 355-366.

123 Castro M, Mathur S, Hargreave F, et al. Reslizumab for poorly controlled, eosinophilic asthma: a randomized, placebo-controlled study. Am J Respir Crit Care Med 2011; 184: 1125-1132.

124 Ibrahim H, O'Sullivan R, Casey D, et al. The effectiveness of reslizumab in severe asthma treatment: a real-world experience. Respir Res 2019; 20: 289.

125 Laviolette M, Gossage DL, Gauvreau G, et al. Effects of benralizumab on airway eosinophils in asthmatic patients with sputum eosinophilia. J Allergy Clin Immunol 2013; 132: 1086-1096.

126 Nair P, Wenzel S, Rabe KF, et al. Oral glucocorticoid-sparing effect of benralizumab in severe asthma. $N$ Engl J Med 2017; 376: 2448-2458

127 Lee HY, Rhee CK, Kang JY, et al. Blockade of IL-33/ST2 ameliorates airway inflammation in a murine model of allergic asthma. Exp Lung Res 2014; 40: 66-76.

128 Ballantyne SJ, Barlow JL, Jolin HE, et al. Blocking IL-25 prevents airway hyperresponsiveness in allergic asthma. J Allergy Clin Immunol 2007; 120: 1324-1331. 